\title{
Sacroplastia como tratamiento del dolor en metástasis sacra
}

\author{
J. Valencia-Anguita; M. Juliá-Narváez; F. Rodríguez-Burgos y A. Ponce de León
}

Servicio de Neurocirugía. Hospital de Traumatología y Rehabilitación. Complejo Hospitales Universitarios Virgen del Rocío. Sevilla.

\section{Resumen}

La inyección o cementación percutánea de polimetil-metacrilato es un tratamiento cada vez de uso más frecuente en el tratamiento del dolor en los pacientes con metástasis vertebrales.

Se presenta el caso de una paciente de 43 años que refería un cuadro de dolor sacro a punta de dedo muy invalidante, secundario a infiltración metastásica de carcinoma de mama a nivel del sacro, con una inmediata y drástica mejoría del dolor tras la cementación percutánea.

PALABRAS CLAVE: Sacroplastia. Vertebroplastia.

Percutaneous sacroplasty for relieving pain caused by sacral metastases

\section{Summary}

Percutaneous injection of polymethyl-metacrylate is an increasingly popular treatment for spine metastatic tumors. We present a case of sacral breast carcinoma treated successfully by percutaneous sacroplasty with injection of bone cement, resulting in pain relief.

KEY WORDS: Sacroplasty. Vertebroplasty.

\section{Introducción}

Las metástasis vertebrales son relativamente frecuentes, pudiendo causar dolor y por tanto, un deterioro de la calidad de vida de los pacientes oncológicos.

La Vertebroplastia es una técnica de inyección vertebral percutánea de polimetil-metacrilato (PMMA), que cada vez está siendo más empleada en la práctica quirúrgica con el objetivo de tratar el dolor y mejorar la calidad de vida del paciente. Es ya conocido su empleo para el tratamiento del dolor en las metástasis vertebrales sin embargo, exis-

Recibido: 16-05-06. Aceptado: 23-08-06 ten escasas referencias respecto a su empleo en metástasis localizadas en el sacro ${ }^{10}$.

Presentamos el caso de una mujer con dolor sacro muy incapacitante, secundario a una infiltración metastásica, tratada mediante cementación percutánea de PMMA, con excelente resultado en el alivio del dolor.

\section{Caso clínico}

Paciente mujer de 43 años de edad con antecedente de carcinoma de mama con múltiples metástasis óseas conocidas, que desarrolla dolor a punta de dedo a nivel de ambas alas sacras que precisa de mórficos, y que le produce una importante incapacidad. La resonancia magnética (RM) mostraba una lesión infiltrante y osteolítica en el sacro, a nivel de $\mathrm{S}_{1}$ y parcial de $\mathrm{L}_{5}$ (Figura 1). La enferma había recibido previamente radioterapia holoespinal, de ahí que el radioterapeuta desestimó la reirradiación del sacro; debido a esto se optó por proponer la cementación de $\mathrm{S}_{1}$.

La paciente fue intervenida en posición de decúbito prono, mediante anestesia local y sedación con propofol, y empleando fluoroscopia biplanar. Se colocaron dos trócares en el sacro, una en cada ala sacra y a continuación se inyectaron a través de las agujas 4cc de cemento óseo, $2 \mathrm{cc}$ por cada una de ellas (70\% de PMMA y 30\% de bario). No hubo complicaciones del procedimiento, siendo la paciente dada de alta hospitalaria en el mismo día de la intervención, sin precisar analgesia alguna. El control de RX y TC mostraron una correcta cementación (Figuras 2 y 3). A los 6 meses del tratamiento persistía la mejoría del dolor.

\section{Discusión}

El tratamiento quirúrgico de las metástasis vertebrales es controvertido; aún así, el cirujano debe contribuir a mantener la calidad de vida del paciente hasta el último momento. Es importante que se decida la modalidad de tratamiento a emplear según la expectativa de vida del

Abreviaturas. PMMA: poli metil-metacrilato. RM: resonancia magnética. TC: tomografía computarizada. 


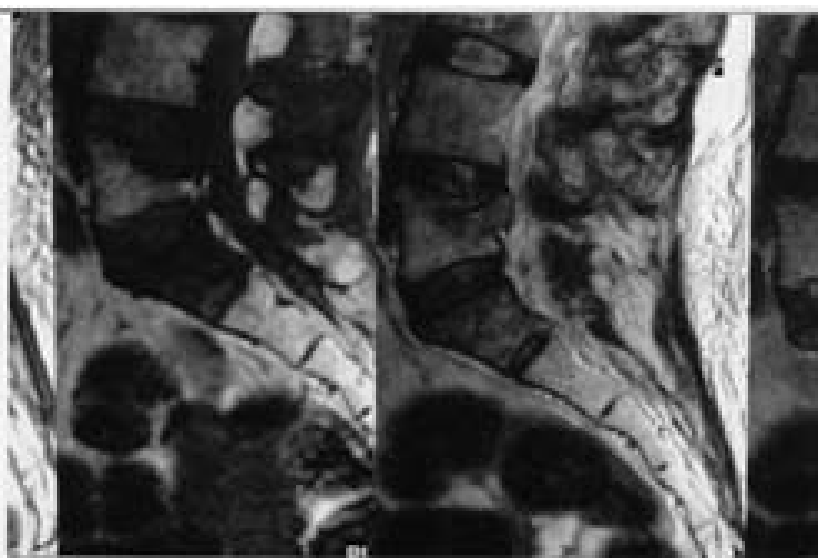

Figura 1. Corte sagital de RM pontenciada en T1 sin contraste mostrando la infiltración neoplásica en S1 y parcial de L5.

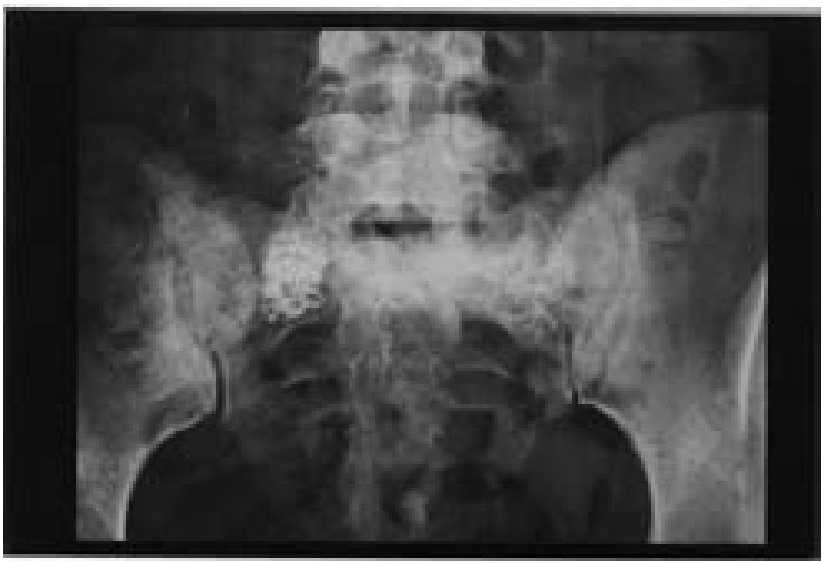

Figura 2. Rx anteroposterior donde se puede observar la ubicación del cemento tras el tratamiento.

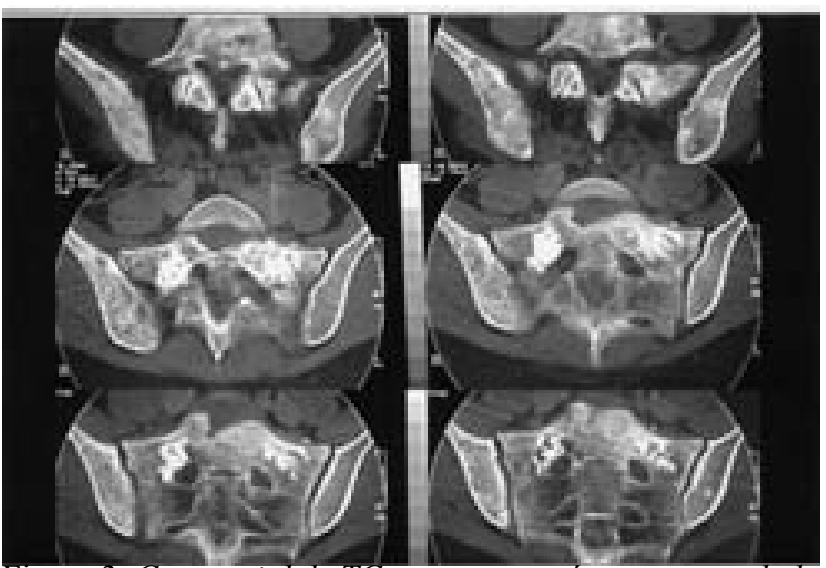

Figura 3. Corte axial de TC con ventana ósea mostrando la localización del cemento en las alas sacras.

enfermo. Hay que reseñar que la mayoría de las metástasis espinales son tratadas con radioterapia, quimioterapia u hormonoterapia. La cirugía está indicada cuando estos tratamientos fracasan, y además el enfermo presenta dolor intratable o déficit neurológico ${ }^{1,9}$.

La vertebroplastia percutánea ha mostrado ser efectiva para el control del dolor de estos pacientes ${ }^{6,7}$, consiguiéndose en la mayoría de ellos controlar el dolor y reducir la dosis de analgésicos, mejorando por tanto la calidad de vida de los mismos. La inyección del PMMA produce una mejoría del dolor por un mecanismo aún desconocido hoy día, tal vez sea por un efecto de estabilización del hueso destruido, además del efecto exotérmico del PMMA inyec$\operatorname{tado}^{10}$. Así mismo, no existe una relación directa entre el alivio del dolor con la cantidad de cemento inyectado según observaron Cotten y cols ${ }^{5}$.

Existen escasas referencias en la bibliografía respecto a la cementación del sacro $^{2,3,4,6,10}$. La mayoría se refieren al tratamiento de la fractura por insuficiencia en pacientes con osteoporosis ${ }^{3,4,6,8}$. Uemura y cols $^{10}$. han utilizado la cementación del sacro en un caso de metástasis de carcinoma hepático, y Atalay y cols ${ }^{2}$, ha empleado la cifoplastia para el tratamiento de un angioma a nivel sacro. Todos estos autores describen buenos resultados para el control del dolor sin complicaciones significativas, En el caso que presentamos también hubo una mejoría inmediata del dolor, que incluso persiste a los 6 meses del tratamiento. Todo esto supone que la cementación del sacro se podría tener en cuenta para el tratamiento del dolor en casos seleccionados con las patologías citadas.

De acuerdo con Pommersheim y cols ${ }^{8}$, las dificultades técnicas de la sacroplastia radican sobre todo en la correcta colocación de los trocares, sobre todo si se emplea la fluoroscopia biplanar. La TC nos ayudaría a solventar el problema de la correcta colocación de la aguja, pero no permite la visualización de la inyección del PMMA a tiempo real como ocurre cuando se emplea la fluoroscopia, y de este modo controlar las fugas del cemento que pudiera ocurrir. Para intentar solventar este problema, Brook y cols ${ }^{3}$, han propuesto la inyección del cemento en el sacro de forma intermitente usando la TC, para que de este modo, se pueda ver cómo se distribuye el PMMA en el sacro durante su inyección, lo cual podría minimizar, en la medida de lo posible, las fugas del cemento.

La evolución a largo plazo de la sacroplastia es desconocida, aún así el resultado descrito por los autores es que se consigue una mejoría sustancial del dolor de los pacientes (igual que en el caso presentado), mejorando de forma importante la calidad de vida de los enfermos. No han ocurrido complicaciones llamativas en todos los casos descritos y tampoco en este caso.

En nuestra opinión la sacroplastia debiera tenerse en cuenta como una opción más de tratamiento en pacientes que presenten dolor intratable secundario a hemangiomas, fractura por insuficiencia y metástasis. 


\section{Bibliografía}

1. Asdourian, P.L.: Metastastatic disease of the spine. In: Bridwell KH, DeWald RL eds. The textbook of spinal surgery, $2^{\text {nd }}$ edition. Philadelphia, PA: Lippincott-Raven Publishers, 1997: 2007-2050.

2. Atalay, B., Caner, H., Yilmaz, C., Altinors, N.: Sacral kyphoplasty for relieving pain caused by sacral hemangioma. Spinal Cord 2006; 44: 196-199.

3. Brook, A.L., Mirsky, D.M., Bello, J.A.: Computerized tomography guided sacroplasty: a practical treatment for sacral insufficiency fracture: case report. Spine 2005; 30: E450.4.

4. Butler, C.L., Given, C.A., Michel, S.J., Tibbs, P.A.: Percutaneous sacroplasty por the treatment of sacral insufficiency fractures. Am J Roentgenol 2005; 184: 1956-1959.

5. Cotten, A., Dewatre, F., Cortet, B., Assaker, R., Leblond, D., Duquesnoy, B.: Percutaneous vertebroplasty for osteolytic metastases and myeloma: Effects or percentage of lesion filling and leakage of methyl-metacrylate at clinical followup. Radiology, 1996: 200: 525-562.

6. Garant, M.: Sacroplasty: a new treatment for sacral insufficiency fracture. J Vasc Intery Radiol 2002; 13: 1265-1267.
7. Kaemmerlen, P., Thiesse, P., Bouvard, H., Biron, P., Mornex, F., Jonas, P.: Percutaneous vetebroplasty in treatment of metastases. Technic and results. J Radiol, 1989; 70: 557562 .

8. Pommersheim, W., Huang-Hellinger, F., Baker, M., Morris, P.: Sacroplasty: a treatment for sacral insufficiency fractures. Am J Neurorradiol 2003; 24: 1003-1007.

9. Tomita, K., Kawahara, N., Kobayashi, T., Yoshida, A., Murakami, H., Akamuru, T.: Surgical strategy for spinal metastases. Spine 2001; 26: 298-306.

10. Uemura, A., Matsusako, M., Numaguchi, Y., Oka, M., Kobayashi, N., Niinami, C., Kawasaki, T., Suzuki, K.: Percutaneous sacroplasty for hemorragia metastases for hepatocelular carcinoma. Am J Neuroradiol 2005; 26: 493 495.

Valencia-Anguita, J.; Juliá-Narváez, M.; RodríguezBurgos, F.; Ponce de León, A.: Sacroplastia como tratamiento del dolor en metástasis sacra. Neurocirugía 2007; 18: 247-249.

Correspondencia postal: Julio Valencia Anguita. Plaza Alfonso de Cossio 7, $5^{\circ}$ D. 41004 Sevilla. 